\title{
Theory of optical-tweezers forces near a plane interface
}

\author{
Dutra, Rafael de Sousa; Neto, P. A. Maia; Nussenzveig, H. M.; Flyvbjerg, Henrik
}

Published in:

Physical Review A (Atomic, Molecular and Optical Physics)

Link to article, DOI:

10.1103/PhysRevA.94.053848

Publication date:

2016

Document Version

Publisher's PDF, also known as Version of record

Link back to DTU Orbit

Citation (APA):

Dutra, R. D. S., Neto, P. A. M., Nussenzveig, H. M., \& Flyvbjerg, H. (2016). Theory of optical-tweezers forces near a plane interface. Physical Review A (Atomic, Molecular and Optical Physics), 94(5), [053848].

https://doi.org/10.1103/PhysRevA.94.053848

\section{General rights}

Copyright and moral rights for the publications made accessible in the public portal are retained by the authors and/or other copyright owners and it is a condition of accessing publications that users recognise and abide by the legal requirements associated with these rights.

- Users may download and print one copy of any publication from the public portal for the purpose of private study or research

- You may not further distribute the material or use it for any profit-making activity or commercial gain

- You may freely distribute the URL identifying the publication in the public portal 


\title{
Theory of optical-tweezers forces near a plane interface
}

\author{
R. S. Dutra, ${ }^{1,2}$ P. A. Maia Neto, ${ }^{3,4}$ H. M. Nussenzveig, ${ }^{3,4}$ and H. Flyvbjerg ${ }^{2}$ \\ ${ }^{1}$ LISComp-IFRJ, Instituto Federal de Educação, Ciência e Tecnologia, Rua Sebastião de Lacerda, Paracambi, RJ, 26600-000, Brazil \\ ${ }^{2}$ Department of Micro-and Nanotechnology, Technical University of Denmark, DK-2800 Lyngby, Denmark \\ ${ }^{3}$ LPO-COPEA, Instituto de Ciências Biomédicas, Universidade Federal do Rio de Janeiro, Rio de Janeiro, RJ, 21941-590, Brazil \\ ${ }^{4}$ Instituto de Física, Universidade Federal do Rio de Janeiro, Caixa Postal 68528, Rio de Janeiro, RJ, 21941-972, Brazil
}

(Received 29 August 2016; published 28 November 2016)

\begin{abstract}
Optical-tweezers experiments in molecular and cell biology often take place near the surface of the microscope slide that defines the bottom of the sample chamber. There, as elsewhere, force measurements require forcecalibrated tweezers. In bulk, one can calculate the tweezers force from first principles, as recently demonstrated. Near the surface of the microscope slide, this absolute calibration method fails because it does not account for reverberations from the slide of the laser beam scattered by the trapped microsphere. Nor does it account for evanescent waves arising from total internal reflection of wide-angle components of the strongly focused beam. In the present work we account for both of these phenomena. We employ Weyl's angular spectrum representation of spherical waves in terms of real and complex rays and derive a fast-converging recursive series of multiple reflections that describes the reverberations, including also evanescent waves. Numerical simulations for typical setup parameters evaluate these effects on the optical force and trap stiffness, with emphasis on axial trapping. Results are in good agreement with available experimental data. Thus, absolute calibration now applies to all situations encountered in practice.
\end{abstract}

DOI: 10.1103/PhysRevA.94.053848

\section{INTRODUCTION}

In optical tweezers (OTs), a highly focused laser beam captures microscopic neutral particles and is used to exert and measure forces and displacements on the micro- and nanoscale. OTs are applied, e.g., in colloid and interface science and in molecular and cell biology [1].

An example in interface science is the measurement of surface interactions between a microsphere and a glass slide [2] or between two microspheres [3,4]. In biological applications, transparent microspheres are usually employed as force transducers, with laser light of wavelength $\lambda \sim 1 \mu \mathrm{m}$ to avoid damage to living cells. An example is the measurement of membrane elastic properties by pulling tethers (nanotubes) from the cell surface [5]. The cell rests on a glass slide that forms the bottom of the sample chamber containing the water that the cell is immersed in.

For not too large displacements from equilibrium, a trapped microsphere experiences a Hookean restoring force. Consequently, force calibration "only" requires determination of the stiffness of the trap. Until recently, this could only be done experimentally and only as a relative calibration: a simultaneous calibration of length scale was required in order to obtain an absolute value for the stiffness [6-9]. Now it can be done by calculating the tweezers force from first principles [10]. For the transverse stiffness, this absolute calibration was achieved subject only to the restriction that the distance between the microsphere surface and the glass slide should not be less than the radius $a$ of the microsphere.

One often has to work closer to the microscope slide than that, however. Both tether-pulling from cells and studies of surface interaction forces are examples of this. In the present work, we address this problem, focusing on the axial stiffness, which is often the relevant one in this context.

The calculation in [10], as well as previous models for the effect of the slide interface [11-13], neglected two effects that are relevant for a microsphere trapped close to the glass slide. One is the total reflection at the glass-water interface of laser beam rays beyond the critical angle and the resulting generation of evanescent waves. The other, and most important one, is the reverberation of light scattered by the microsphere between its surface and the glass slide, as demonstrated experimentally in Refs. [14-16].

Absolute calibration theory requires an accurate description of the wide-angle laser beam incident on the microsphere and of its scattering. Both are problems in diffraction theory. The exact solution to the scattering problem is given by the Mie partial-wave series [17]. In scalar scattering, an exact representation of a converging beam in free space as an angular spectrum of plane waves was given by Debye [18] Its electromagnetic generalization is due to Richards and Wolf [19]. It is also indispensable for absolute calibration to take account of aberration effects. One of them is interface spherical aberration, the defocusing effect of refraction of the laser beam at the interface between the glass slide and the water in the sample chamber. By including also all remaining primary aberrations of the optical system, one gets the so-called MDSA+ result (Mie-Debye-interface spherical aberration + other primary aberrations) [10].

A seminal treatment of reverberation effects for the scattering of a plane wave by a sphere above a plane substrate was given by Bobbert and Vlieger [20]. It is based on Weyl's beautiful formula $[21,22]$ representing spherical waves as an angular spectrum of plane waves propagating in real and complex directions [23], tracking reflections and refractions of these waves at the plane interface. It provides another illustration of the role of analytic continuation in diffraction theory, which can be traced to the principle of causality [24].

The same problem was also treated by Videen [25] and by Wriedt and Doicu [26], assuming near-normal incidence. In [27], an evanescent mode, excited in a prism, is multiply scattered by a microsphere located above it. A similar situation 


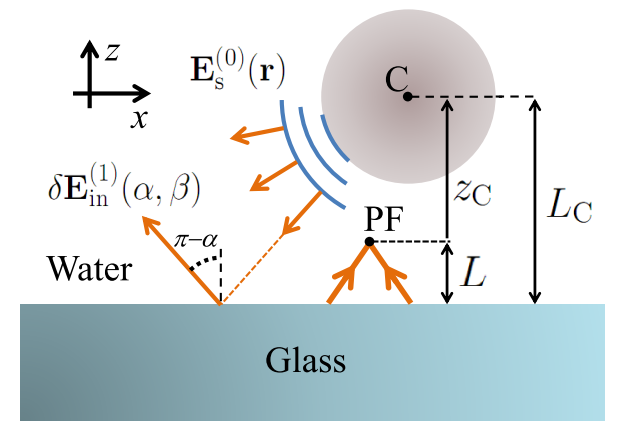

FIG. 1. Microsphere immersed in water, with center C distant $L_{\mathrm{C}}$ from glass slide. $\mathrm{PF}=$ paraxial focus of incident laser beam. $\mathbf{E}_{\mathrm{s}}^{(0)}=$ zero-order spherical wave; $\delta \mathbf{E}_{\text {in }}^{(1)}(\alpha, \beta)=$ first-order of iteration for component of incident angular spectrum in $(\alpha, \beta)$ direction.

is treated in [28], with an evanescent mode produced in a waveguide. None of the above models, however, describe the situation found in an OT setup.

In the present work we develop an iterative method that treats reverberation in OT setups as an extension of the MDSA+ solution. Section II presents the general formalism and the derivation of optical force corrections. Section III gives numerical results. Section IV compares our theoretical results with experimental results from Ref. [15]. Section V concludes. Some technical points have been relegated to Appendixes A and $\mathrm{B}$.

\section{GENERAL FORMALISM}

In this section we describe the principal steps in the derivation of the optical force. We account for multiple reflections of light between the microsphere and the glass slide, resulting in an iterative approach in terms of scattering order at the glass surface. This is similar to the treatment of interference effects in plane parallel plates: they also may be treated as boundary-value problems or, equivalently, by summation of a series of multiple reflections [29]. An analogous approach was employed in [30,31].

The setup is represented in Fig. 1. The center $\mathrm{C}$ of the microsphere is located at a distance $L_{\mathrm{C}}$ from the glass slide. We choose the paraxial focus PF as origin of coordinates. It is located on the optical axis at a distance $L$ from the surface of the glass slide. Thus the position vector $\mathbf{r}_{\mathrm{C}}$ for $\mathrm{C}$ is, in cylindrical coordinates, $\left(\rho_{\mathrm{C}}, \phi_{\mathrm{C}}, z_{\mathrm{C}}\right)$ with $z_{\mathrm{C}}=L_{\mathrm{C}}-L$.

The circularly polarized Gaussian incident beam travels along the $z$ direction with helicity $\sigma^{+}$, vacuum wavelength $\lambda_{0}$, and waist $w_{0}$ at the objective entrance port (radius $R_{0}$ ). It is focused by an objective with high numerical aperture NA. Its electric field, beyond the glass-water interface, is given by the Debye-type angular spectrum representation [19]:

$$
\begin{aligned}
\mathbf{E}_{\mathrm{in}}^{(0)}(\mathbf{r})= & E_{0} \int_{0}^{2 \pi} d \varphi \int_{0}^{\vartheta_{m}} d \vartheta \sin \vartheta \sqrt{\cos \vartheta} e^{-\gamma^{2} \sin ^{2} \vartheta} \\
& \times T(\vartheta) e^{i\left[\Phi_{\mathrm{g}-\mathrm{w}}(\vartheta)+\Phi_{\text {add }}(\vartheta, \varphi)\right]} e^{i \mathbf{k}_{\mathrm{w}} \cdot\left(\mathbf{r}+\mathbf{r}_{a}\right)} \hat{\mathbf{x}}^{\prime}\left(\vartheta_{\mathrm{w}}, \varphi_{\mathrm{w}}\right),
\end{aligned}
$$

with $\vartheta_{m}=\sin ^{-1}\left(\mathrm{NA} / n_{\mathrm{g}}\right) \quad$ representing the aperture angle in the glass medium (refractive index $n_{\mathrm{g}}$ ) and
$\gamma=R_{0} /\left(w_{0} \sin \vartheta_{m}\right)$. Each plane wave in the water medium (refractive index $n_{\mathrm{w}}$ ) propagates with wave vector $\mathbf{k}_{\mathrm{w}}\left(\vartheta_{\mathrm{w}}, \varphi_{\mathrm{w}}\right)$ with $k_{\mathrm{w}}=2 \pi n_{\mathrm{w}} / \lambda_{0}$ and polar angles $\varphi_{\mathrm{w}}=\varphi$ and $\vartheta_{\mathrm{w}}=\sin ^{-1}(\sin \vartheta / N)$, with $N=n_{\mathrm{w}} / n_{\mathrm{g}}$. The amplitude is multiplied by the Fresnel transmission coefficient

$$
T(\vartheta)=\frac{2 \cos \vartheta}{\cos \vartheta+N \cos \vartheta_{\mathrm{w}}} .
$$

It also acquires a phase factor given by the sum of the function $[13,32]$

$$
\Phi_{\mathrm{g}-\mathrm{w}}(\vartheta)=k\left(-\frac{L}{N} \cos \vartheta+N L \cos \vartheta_{\mathrm{w}}\right),
$$

representing the spherical aberration introduced by the planar interface, and $\Phi_{\text {add }}(\vartheta, \varphi)$ due to additional primary optical aberrations present in the setup [10].

When NA $>n_{\mathrm{w}}$, rays incident at the glass-water interface at angles larger than the critical angle, $\vartheta>\sin ^{-1} N$, undergo total internal reflection, giving rise to evanescent waves. They are included in Eq. (1) since $\vartheta_{\mathrm{w}}$ becomes complex in this case.

The iterative solution results from the summation of the series of multiple reflections [29] for the incident electric field

$$
\mathbf{E}_{\text {in }}=\mathbf{E}_{\text {in }}^{(0)}+\delta \mathbf{E}_{\text {in }}^{(1)}+\delta \mathbf{E}_{\text {in }}^{(2)} \ldots,
$$

with $\delta \mathbf{E}_{\mathrm{in}}^{(k)}$ containing $k$ reflections by the glass slide, and corresponding scattered fields

$$
\mathbf{E}_{\mathrm{s}}=\mathbf{E}_{\mathrm{s}}^{(0)}+\delta \mathbf{E}_{\mathrm{s}}^{(1)}+\delta \mathbf{E}_{\mathrm{s}}^{(2)} \cdots
$$

Here the zero-order scattered field $\mathbf{E}_{\mathrm{s}}^{(0)}$ corresponds to the situation where we consider only scattering by the microsphere with no reflection at the surface of the glass slide, i.e., the situation described by the MDSA+ theory [10].

How fast do we expect the multiple reflection series to converge? In an alternative approach to the solution, employing the method of images [25], it was shown that the angle of incidence of the zero-order scattered field on the interface does not exceed $30^{\circ}$, the value it has for a microsphere resting on the glass slide. The corresponding Fresnel reflectivity for a water-glass interface is less than $0.3 \%$. Therefore, the first-order reflected beam intensity is very weak, and it is nearly paraxial close to the interface. Consequently, it is expected to exert chiefly an upward radiation pressure, leading to a repulsive axial force in this region. Interference between the first- and zero-order scattered fields gives rise to a sinusoidal modulation, with a contrast roughly proportional to $\left|\delta \mathbf{E}_{\mathrm{s}}^{(1)}\right| /\left|\mathbf{E}_{\mathrm{s}}^{(0)}\right|$. One can derive an upper bound by considering the case of parallel planar water-glass interfaces, for which the contrast is $\sim 5 \%$. These semiquantitative estimates indicate that the iteration method is rapidly convergent.

The key theoretical tool used to develop the iterative method is expansion of the field backscattered by the microsphere as a superposition of plane waves. We first introduce the complex angular spectrum representation [33]

$$
\mathbf{E}(\mathbf{r})=\frac{i k_{\mathrm{w}}}{2 \pi} \int_{0}^{2 \pi} d \beta \int_{C} d \alpha \sin \alpha \mathbf{E}(\alpha, \beta) e^{i \mathbf{k}_{\mathrm{w}} \cdot \mathbf{r}}
$$

and

$$
\mathbf{H}(\mathbf{r})=\frac{i k_{\mathrm{w}}}{2 \pi} \int_{0}^{2 \pi} d \beta \int_{C} d \alpha \sin \alpha \mathbf{H}(\alpha, \beta) e^{i \mathbf{k}_{\mathrm{w}} \cdot \mathbf{r}},
$$


with $\mathbf{k}_{\mathrm{w}}=\mathbf{k}_{\mathrm{w}}(\alpha, \beta)$ given in terms of the spherical angles $\alpha$ and $\beta$. For fields propagating downward, the path $C$ is a vertical line in the complex plane from $\pi / 2+i \infty$ to $\pi / 2$, joined by the interval from $\pi / 2$ to $\pi$ along the real axis. The integration path is chosen so as to take into account both the homogeneous and inhomogeneous (imaginary values of $\alpha$ ) plane waves that propagate back to the glass surface.

In addition to the angular spectrum representation, it is also convenient to multipole-expand the fields [34] when solving the Mie scattering problem at each iteration. Each plane-wave component is written in terms of electric $\left(\Pi^{\mathrm{E}}\right)$ and magnetic $\left(\Pi^{\mathrm{M}}\right)$ Debye potentials:

$$
\begin{aligned}
\mathbf{E}(\alpha, \beta)= & k_{\mathrm{w}} \hat{\mathbf{k}} \times \mathbf{L} \Pi^{\mathrm{E}}(\alpha, \beta) \\
& +\sqrt{\frac{\mu_{0}}{\varepsilon_{\mathrm{w}}}} k_{\mathrm{w}} \mathbf{L} \Pi^{\mathrm{M}}(\alpha, \beta)
\end{aligned}
$$

and

$$
\mathbf{H}(\alpha, \beta)=-\sqrt{\frac{\varepsilon_{\mathrm{w}}}{\mu_{0}}} k_{\mathrm{w}} \mathbf{L} \Pi^{\mathrm{E}}(\alpha, \beta)+k_{\mathrm{w}} \hat{\mathbf{k}} \times \mathbf{L} \Pi^{\mathrm{M}}(\alpha, \beta),
$$

where the orbital angular momentum operator, represented in momentum space, is $\mathbf{L}=-i \hat{\mathbf{k}} \times \nabla_{\hat{\mathbf{k}}}$ and $\varepsilon_{\mathrm{W}}$ represents the medium electric permittivity. We assume the medium to be nonmagnetic, and $\mu_{0}$ is the vacuum permeability.

We now write the electric Debye potential in real space associated with the zero-order incident wave $\mathbf{E}_{\text {in }}^{(0)}$, given by Eq. (1). Its multipole expansion at position $\mathbf{r}(r, \theta, \phi)$ is given by

$$
\Pi_{\mathrm{in}}^{\mathrm{E}(0)}(\mathbf{r})=\frac{i E_{0}}{k_{\mathrm{w}}} \sum_{J M} \gamma_{J M} j_{J}\left(k_{\mathrm{w}} r\right) Y_{J M}(\theta, \phi),
$$

where $j_{J}$ and $Y_{J M}$ denote the spherical Bessel functions and the spherical harmonics [35], respectively. The coefficients $\gamma_{J M}$ are given by (A3) in Appendix A and

$$
\sum_{J M} \equiv \sum_{J=1}^{\infty} \sum_{M=-J}^{J}
$$

The electric Debye potential for the corresponding scattered field follows from the Mie solution:

$$
\Pi_{\mathrm{s}}^{\mathrm{E}(0)}(\mathbf{r})=\frac{i E_{0}}{k_{\mathrm{w}}} \sum_{J M} \gamma_{J M}\left(-a_{J}\right) h_{J}^{(1)}\left(k_{\mathrm{w}} r\right) Y_{J M}(\theta, \phi),
$$

where $a_{J}$ are the electric multipole Mie coefficients [36] and the spherical Hankel functions of the first kind $h_{J}^{(1)}\left(k_{\mathrm{w}} r\right)$ [35] represent outgoing spherical waves. To describe the interaction of the Mie scattered wave with the glass-water interface, we need to expand the scattered spherical waves into plane waves. For this, we follow [20,21] by employing the integral Weyl representation for the product of spherical Hankel and spherical harmonics functions $\Pi_{J M}(\mathbf{r}) \equiv h_{J}^{(1)}\left(k_{\mathrm{w}} r\right) Y_{J M}(\theta, \phi)$

$$
\Pi_{J M}(\mathbf{r})=\frac{i^{-J}}{2 \pi} \int_{0}^{2 \pi} d \beta \int_{C} d \alpha \sin \alpha Y_{J M}(\alpha, \beta) e^{i \mathbf{k}_{\mathrm{w}} \cdot \mathbf{r}}
$$

Substituting (12) into (11), we find a spectral decomposition for the scattered Debye potential, given by

$$
\begin{aligned}
\Pi_{\mathrm{s}}^{\mathrm{E}(0)}(\mathbf{r})= & -\frac{i E_{0}}{2 \pi k_{\mathrm{w}}} \sum_{J M}(-i)^{J} \gamma_{J M} a_{J} \\
& \times \int_{0}^{2 \pi} d \beta \int_{C} d \alpha \sin \alpha Y_{J M}(\alpha, \beta) e^{i \mathbf{k}_{\mathrm{w}} \cdot \mathbf{r}} .
\end{aligned}
$$

By analogy with Eq. (6), this leads to the following spectral amplitude for the electric Debye potential:

$$
\Pi_{\mathrm{s}}^{\mathrm{E}(0)}(\alpha, \beta)=-\frac{E_{0}}{k_{\mathrm{w}}^{2}} \sum_{J M}(-i)^{J} \gamma_{J M} a_{J} Y_{J M}(\alpha, \beta) .
$$

By an analogous procedure, we obtain a similar amplitude for the magnetic Debye potential. We substitute (14) into (8) in order to find the angular spectrum representation of the zeroorder scattered field. In this representation, it is straightforward to consider the reflection by the planar glass surface. The result is in its turn replaced into (6), leading to the following expansion for the electric field in real space after one iteration:

$$
\begin{aligned}
\delta \mathbf{E}_{\mathrm{in}}^{(1)}(\mathbf{r})= & -\frac{E_{0}}{4 \pi} \sum_{J M} i^{-J} \sqrt{\frac{J(J+1)(2 J+1)}{4 \pi}} \gamma_{J M} \\
& \times \int_{0}^{2 \pi} d \beta \int_{C} d \alpha \sin \alpha e^{i M \beta} e^{-2 i k_{\mathrm{w}} L_{\mathrm{C}} \cos \alpha} \\
& \times\left[\left(b_{J}+a_{J}\right)\left(r_{\mathrm{TM}} \hat{\vartheta}+i r_{\mathrm{TE}} \hat{\varphi}\right) d_{M, 1}^{J}(\alpha)\right. \\
& \left.+\left(b_{J}-a_{J}\right)\left(r_{\mathrm{TM}} \hat{\vartheta}-i r_{\mathrm{TE}} \hat{\varphi}\right) d_{M,-1}^{J}(\alpha)\right] e^{i \mathbf{k}_{\mathrm{w}}(\pi-\alpha, \beta) \cdot \mathbf{r}},
\end{aligned}
$$

where $b_{J}$ are the magnetic multipole Mie coefficients [36] and $d_{M, \pm 1}^{J}(\alpha)$ are the matrix elements of finite rotations [37]. The unit vectors $\hat{\vartheta}$ and $\hat{\varphi}$ are calculated in the direction $(\pi-\alpha, \beta)$ in Fourier space. In (15), the electric field is written as a sum of transverse electric (TE) and transverse magnetic (TM) contributions, each one multiplied by the corresponding Fresnel coefficients $r_{\mathrm{TE}}$ and $r_{\mathrm{TM}}$, both calculated for the incidence angle $\pi-\alpha$ (see Fig. 1). The phase factor $\exp \left(-2 i k_{\mathrm{w}} L_{\mathrm{C}} \cos \alpha\right)$ accounts for the double path of light between microsphere and glass slide. The result (15) represents the first-order iteration, arising from one scattering by the microsphere and one reflection at the glass surface.

The incident and scattered electric fields describing the interaction between the microsphere and the glass surface can be written as

$$
\mathbf{E}_{\mathrm{in}}=\mathbf{E}_{\mathrm{in}}^{(0)}+\delta \mathbf{E}_{\mathrm{in}},
$$

and

$$
\mathbf{E}_{\mathrm{s}}=\mathbf{E}_{\mathrm{s}}^{(0)}+\delta \mathbf{E}_{\mathrm{s}}
$$

where $\delta \mathbf{E}_{\mathrm{in}}=\sum_{k} \delta \mathbf{E}_{\mathrm{in}}^{(k)}$ and $\delta \mathbf{E}_{\mathrm{s}}=\sum_{k} \delta \mathbf{E}_{\mathrm{s}}^{(k)}$ are the contributions arising from the glass surface. They are described by the multiple reflections series derived in Appendix A. The sum $\sum_{k} \equiv \sum_{k=1}^{n}$ represents the effect of $n$ multiple reflections on the glass surface, where the number of reflections $n$ determines the iteration order.

The optical force is calculated using the Maxwell stress tensor formalism following the same steps as in previous 
works [10,11]. As the Maxwell stress tensor is quadratic over the electromagnetic fields, we find two different contributions after substituting the total electric field $\mathbf{E}=\mathbf{E}_{\text {in }}+\mathbf{E}_{\mathrm{s}}$; there is extinction, associated with the cross term $\mathbf{E}_{\mathrm{in}} \cdot \mathbf{E}_{\mathrm{s}}^{*}$, and there is scattering, coming from $\mathbf{E}_{\mathrm{s}} \cdot \mathbf{E}_{\mathrm{s}}^{*}$. The final result is written in terms of the dimensionless optical force efficiency

$$
\mathbf{Q}=\frac{\mathbf{F}}{n_{\mathrm{w}} P / c},
$$

where $P$ is the local power at the sample chamber, $c$ is the speed of light in vacuum, and $\mathbf{F}$ is the optical force. The iterative corrections to extinction come from $\operatorname{Re}\left(\mathbf{E}_{\text {in }}^{(0)} \cdot \delta \mathbf{E}_{\mathrm{s}}^{*}\right)$ and $\operatorname{Re}\left(\delta \mathbf{E}_{\text {in }} \cdot \mathbf{E}_{\mathrm{s}}^{*}\right)$, leading to the extinction terms $\delta \mathbf{Q}_{\mathrm{e}}^{\mathrm{i}}$ and $\delta \mathbf{Q}_{\mathrm{e}}^{\mathrm{ii}}$, respectively. For the scattering contribution, we have the correction $\delta \mathbf{E}_{\mathrm{s}} \cdot \delta \mathbf{E}_{\mathrm{s}}^{*}+2 \operatorname{Re}\left(\mathbf{E}_{\mathrm{s}}^{(0)} \cdot \delta \mathbf{E}_{\mathrm{s}}^{*}\right)$, where the second term in the sum, representing the interference between the zero-order and corrected scattered fields, is the dominant contribution. The trap efficiencies associated with the extinction and scattering terms, respectively, are then written as

$$
\mathbf{Q}_{\mathrm{e}}=\mathbf{Q}_{\mathrm{e}}^{(0)}+\delta \mathbf{Q}_{\mathrm{e}}^{\mathrm{i}}+\delta \mathbf{Q}_{\mathrm{e}}^{\mathrm{ii}}
$$

and

$$
\mathbf{Q}_{\mathrm{s}}=\mathbf{Q}_{\mathrm{s}}^{(0)}+\delta \mathbf{Q}_{\mathrm{s}},
$$

where $\mathbf{Q}_{\mathrm{e}}^{(0)}$ and $\mathbf{Q}_{\mathrm{s}}^{(0)}$ represent the optical force efficiencies in zero order (MDSA+ theory) and the total efficiency is given by $\mathbf{Q}=\mathbf{Q}_{\mathrm{e}}+\mathbf{Q}_{\mathrm{s}}$. In Appendix B we write the explicit expressions for the efficiency factor terms $\mathbf{Q}_{\mathrm{s}}$ and $\mathbf{Q}_{\mathrm{e}}$ including the corrections due to multiple reflections.

\section{AXIAL FORCE AND INTERFERENCE OSCILLATIONS}

For the numerical results presented in this paper [38], we take the following parameters, representative of typical OT experiments: laser wavelength $\lambda_{0}=1.064 \mu \mathrm{m}$; laser beam waist $w_{0}=5.4 \mathrm{~mm}$ at the objective entrance of radius $R_{0}=$ $3.22 \mathrm{~mm}$; glass, water, and polystyrene microsphere refractive indexes $n_{\mathrm{g}}=1.51, n_{\mathrm{w}}=1.332$, and $n_{\mathrm{b}}=1.576+i 0.001$, respectively.

The reverberation in MDSA+ theory causes interference between the incident and the backscattered fields, which generates stationary waves inside the cavity between the microsphere and the glass surface. The first term in the series of multiple reflections is sufficient to guarantee a result correct to three significant digits. Below, we include three terms $(n=3)$, which yields a precision of six significant digits. This confirms our estimate that the iteration series converges fast for the relative refractive indexes $N=n_{\mathrm{w}} / n_{\mathrm{g}}$ and $n_{\mathrm{b}} / n_{\mathrm{w}}$ close to one in typical OT setups.

Figure 2 shows the dimensionless axial force efficiency $Q_{z}$ as function of the height $L_{\mathrm{C}}$ of the center of the microsphere over the glass slide. Numerical results are shown for three different numerical apertures, $\mathrm{NA}=1.0,1.25$, and 1.4. We take the microsphere radius $a=1.0 \mu \mathrm{m}$ and fix the paraxial focal position at the height $L=2 \mu \mathrm{m}$ above the glass slide. Objectives with higher numerical aperture produce beams with a larger opening angle, increasing the effect of the gradient force over radiation pressure, thereby increasing the counterpropagating trapping force (well depth) toward the glass slide. More importantly, the interference between the incident

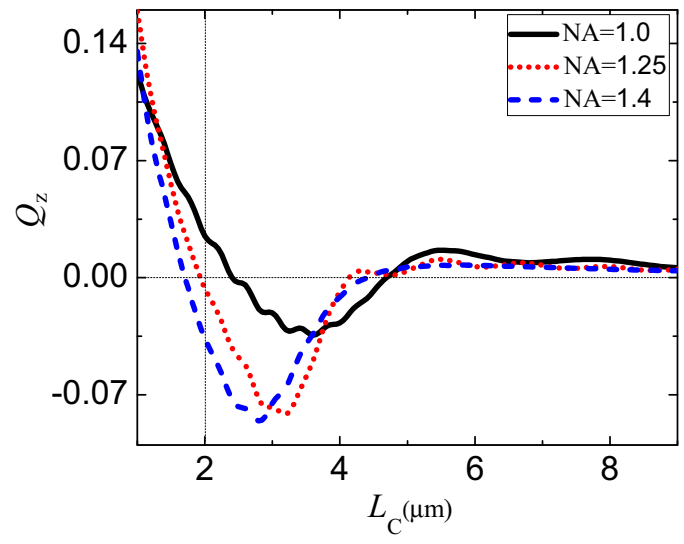

FIG. 2. Dimensionless axial force efficiency $Q_{z}$ for a microsphere with radius $a=1.0 \mu \mathrm{m}$ as a function of the height $L_{\mathrm{C}} \geqslant a$ of the center of the microsphere over the surface of the glass slide. Results are shown for three different numerical apertures (NA) of the objective, all for the same paraxial focal position, fixed at height $L=2.0 \mu \mathrm{m}$ over the surface of the glass slide, as indicated by the vertical dotted line.

beam and the backscattered light is seen as a periodic modulation of $Q_{z}$ as function of $L_{\mathrm{C}}$. The modulation period is approximately one-half of the laser wavelength in water, $\lambda_{\mathrm{w}} / 2 \approx 0.4 \mu \mathrm{m}$, as in a standard Fabry-Perot interferometer, and in agreement with experimental observations of the scattered intensity by a trapped sphere close to the glass slide [15]. Differently from an interferometer with parallel planar interfaces, the modulation amplitude decreases with increasing distance between sphere and glass surface. This is expected since the amplitude of the field backscattered by the sphere decays with distance, and hence its reflection by the glass slide decreases with $L_{\mathrm{C}}$. In agreement with the experiment reported in Ref. [14], Fig. 2 also indicates that the reverberation effect is stronger for the smaller numerical apertures, which correspond to more collimated incident beams, thus optimizing the multiple scattering between the two surfaces.

In Fig. 3, we plot the axial force efficiency $Q_{z}$ again as a function of the microsphere height $L_{\mathrm{C}}$, for four different values of the microsphere radius: $a=0.5,1.0,1.5$, and $2.0 \mu \mathrm{m}$. We take NA $=1.4$ and the paraxial focus at height $L=2 a$ from the glass slide.

The interference oscillations are most clearly seen for the radii $a=1.0 \mu \mathrm{m}$ and $a=1.5 \mu \mathrm{m}$. Recent interest in axial optical trapping [39] considers a Hookean trapping force in a region around the beam focus. Our results shown in Fig. 3 for $a=1.0 \mu \mathrm{m}$ and $1.5 \mu \mathrm{m}$ indicate the appearance of nonlinearity in this region for microsphere radii slightly larger than or comparable with $\lambda_{\mathrm{w}}$.

Averaging the interference pattern over the microsphere volume leads to a reduction of the modulation amplitude for largest spheres, as illustrated by the case $a=2.0 \mu \mathrm{m}$ in Fig. 3 . Another effect contributing to the reduction of reverberation is the spherical aberration introduced by refraction at the glass-water interface, which increases with the height $L$ of the paraxial focus with respect to the slide. Optical aberrations in general tend to degrade the reverberation effect. In addition 


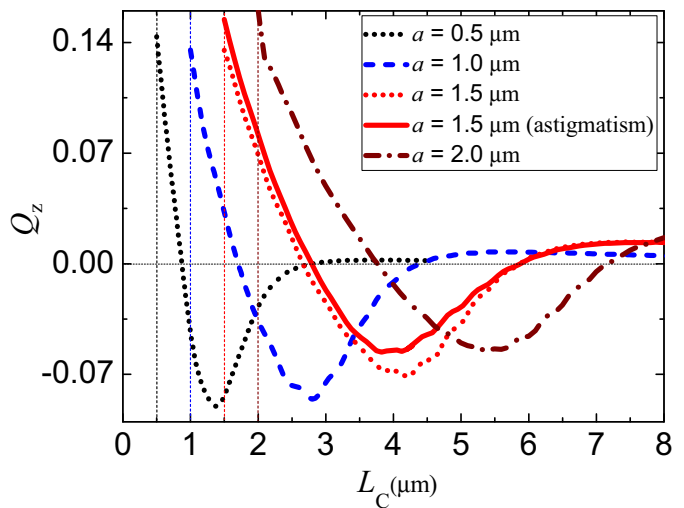

FIG. 3. Dimensionless axial force efficiency $Q_{z}$ as a function of the microsphere height $L_{\mathrm{C}}$ for four different values of the microsphere radius. We take NA $=1.4$ and the paraxial focal position at $L=2 a$ above the glass slide. The vertical dashed lines indicate the position $L_{\mathrm{C}}=a$ of the center of the microsphere when the microsphere touches the surface of the glass slide. The solid line represents the case of a typical astigmatic incident beam (see text), for the radius $a=1.5 \mu \mathrm{m}$.

to the interface spherical aberration already taken into account in all examples considered here, we add, for the solid line ( $a=$ $1.5 \mu \mathrm{m})$ shown in Fig. 3, a moderate amount of astigmatism, corresponding to the Seidel amplitude $A_{\text {ast }}=1.12$ measured for a typical OT setup [10].

For the smallest microsphere shown in Fig. 3, $a=0.5 \mu \mathrm{m}$, the modulation is also too small to be visible in the plot. This is expected since the amplitude of the field scattered by the microsphere decreases as the radius becomes smaller than the wavelength $\lambda_{\mathrm{w}}$. However, the reverberation effect is important as far as the trap stiffness is concerned even for this small microsphere radius, as discussed in the next section.

\section{AXIAL TRAP STIFFNESS}

The axial trap stiffness is obtained by taking the numerical derivative of the axial force efficiency,

$$
k_{z}=-\left.\left(\frac{n_{\mathrm{w}} P}{c}\right) \frac{\partial Q_{z}}{\partial z_{\mathrm{C}}}\right|_{z_{\mathrm{C}}=z_{\mathrm{eq}}},
$$

at the equilibrium position $z_{\text {eq }}$. When using (20), we simulate an actual calibration experiment and take only experimentally known parameters as input values [10]. We start with the microsphere touching the glass slide $\left(L_{\mathrm{C}}=a\right)$ and determine the corresponding focal height $L_{0}$ above the glass slide by solving $Q_{z}\left(a-L_{0}\right)=0$ numerically for $L_{0}$. After performing an upward displacement $d$ of the objective, the corresponding focal height is $L=L_{0}+N d$ (see Fig. 1), and the equilibrium position $z_{\text {eq }}$ of the microsphere is obtained by solving $Q_{z}\left(z_{\text {eq }}\right)=0$ numerically.

For comparison with available experimental data [15], we plot in Fig. 4 the axial trap stiffness per unit power, $k_{z} / P$, as a function of the objective displacement $d$ (circles). In order to find agreement with Fig. 7 of Ref. [15], we add a small amount of spherical aberration, with Seidel amplitude $A_{\mathrm{sa}}=-0.5$, in addition to the spherical aberration introduced by refraction through the glass slide surface. We consider a microsphere

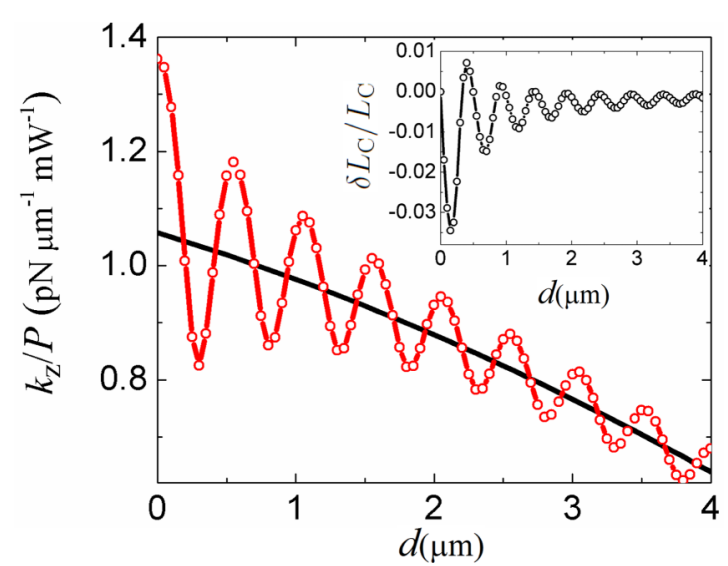

FIG. 4. Axial trap stiffness, normalized by the laser power in the sample chamber, as a function of the objective upward displacement $d$, for a microsphere of $0.5 \mu \mathrm{m}$ radius and an objective with numerical aperture $\mathrm{NA}=1.25$. The value $d=0$ corresponds to the configuration where the microsphere in equilibrium is just touching the glass slide. Solid line: MDSA+ theory. Circles: MDSA+ theory corrected for the effect of reverberation. The inset shows the relative correction of the axial equilibrium position, as a function of the objective displacement.

with radius $a=0.5 \mu \mathrm{m}$ and an objective with numerical aperture $\mathrm{NA}=1.25$, with other parameter values as listed at the beginning of the previous section. For comparison, the result of MDSA + theory without accounting for reverberation is also shown (solid line).

With the microsphere close to the glass slide, reverberation matters most: the numerical values oscillate around the MDSA+ curve with an amplitude of $30 \%$ for small objective displacements. The modulation period is again consistent with the value $\lambda_{\mathrm{w}} / 2$ expected for stationary waves as found in the previous section. In fact, the period $\Delta d$ obtained from Fig. 4 is such that $\Delta L=N \Delta d \approx 0.4 \mu \mathrm{m}$.

For increasing values of $d$, the amplitude of the oscillations decreases and MDSA+ is recovered. The spherical aberration introduced by refraction at the glass-water interface degrades the focal region [32] and as consequence reduces the axial trap stiffness. This effect increases with the objective displacement $d$, thus explaining the steady decay of the MDSA+ curve with $d$.

Experimental results for the axial trap stiffness variation with the vertical displacement of the microscope stage were reported in Ref. [15] for a bead radius $a=0.545 \mu \mathrm{m}$ and $\mathrm{NA}=1.25$. Figure 7 of Ref. [15] presents all the features discussed here in connection with Fig. 4: oscillations with a decaying amplitude around an average value, corresponding to the MDSA + curve, that decreases with distance from the glass slide. The period and amplitude of oscillations are also consistent with our numerical results.

The inset of Fig. 4 shows the relative correction of the axial equilibrium position, $\left(L_{\mathrm{C}}-L_{\mathrm{C}}^{\mathrm{MDSA}}\right) / L_{\mathrm{C}}$, as a function of the objective displacement $d$, with $d=0$ corresponding to the microsphere touching the glass slide in equilibrium $\left(L_{\mathrm{C}}=L_{\mathrm{C}}^{\mathrm{MDSA}}=a\right)$. For a given focal height, reverberation brings the equilibrium position slightly closer to the glass 


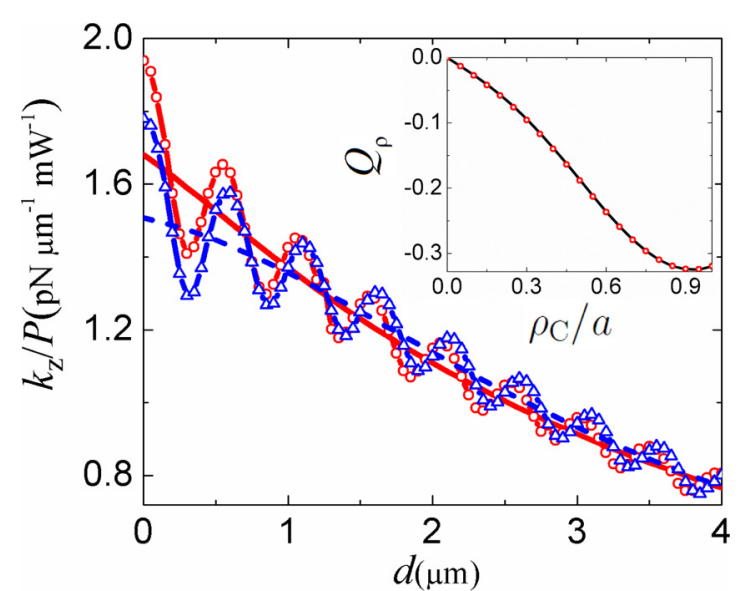

FIG. 5. Axial trap stiffness, normalized by the laser power in the sample chamber, as a function of objective displacement $d$, for a microsphere of $0.5 \mu \mathrm{m}$ radius and an objective with numerical aperture NA $=1.4$. Solid line: MDSA+ theory taking into account the evanescent waves. Dashed line: MDSA+ theory cutting off the evanescent waves, for an effective numerical aperture NA $=1.332$. Circles: MDSA+ theory taking into account the evanescent waves as well as the reverberation from the glass surface. Triangles: MDSA+ theory without evanescent waves taking into account the reverberation from the glass surface. Inset: dimensionless transverse force efficiency as a function of radial position, in units of microsphere radius, for an objective displacement $d=0$. Solid line: MDSA+ theory taking into account the evanescent waves. Circles: MDSA+ theory taking into account the evanescent waves and the reverberation from the glass surface.

slide. The oscillations of the equilibrium position have a small amplitude and were not detected in Ref. [15].

Finally, we discuss the effect of the inclusion of evanescent waves on the axial trap stiffness, by comparing results with and without this inclusion. We consider a microsphere of $0.5 \mu \mathrm{m}$ radius and an objective with numerical aperture NA $=1.4>$ $n_{\mathrm{w}}$, giving a maximum aperture angle of the laser beam in glass larger than the critical angle $\theta_{c}=\arcsin \left(n_{\mathrm{w}} / n_{\mathrm{g}}\right)$. In this situation, the angle $\theta_{\mathrm{w}}$ of propagation inside water becomes complex when the corresponding angle $\theta_{\mathrm{g}}$ in glass is larger than the critical angle $\theta_{c}$, giving rise to evanescent waves. In Fig. 5, we plot the axial trap stiffness, normalized by the laser power in the sample chamber, as a function of the objective displacement $d$, for a microsphere of $0.5 \mu \mathrm{m}$ radius and an objective with numerical aperture $\mathrm{NA}=1.4$, comparing four different theories: (i) MDSA+ theory including evanescent waves (solid line); (ii) MDSA+ theory simulating the use of a diaphragm to cut off the evanescent waves, with an effective NA $=1.332=n_{\mathrm{w}}$ (dashed line). (iii) Same as (i), including reverberation (circles). (iv) Same as (ii), including reverberation (triangles). In both (iii) and (iv), the curves oscillate around the respective curves without reverberation, with the period and decaying amplitude as described in connection with Fig. 4. The vertical shift between the curves for small displacements arises from the effect of evanescent waves reinforcing the gradient force and the consequent increase of the axial stiffness. On the other hand, for large objective displacements $d$, the focal position is too far from the glass slide, and the contribution of evanescent waves is negligible, as expected.

The inset of Fig. 5 shows the dimensionless transverse force efficiency for objective displacement $d=0$, which maximizes reverberation effects on the axial trap stiffness, with (circles) and without reverberation (solid). The evanescent waves were accounted for in these results. We see that reverberation does not play an important role for the transverse force and consequently neither for the transverse trap stiffness.

\section{CONCLUSION}

In the present work, the behavior of OT force components in the neighborhood of the glass slide for a typical OT setup was addressed theoretically with an iterative approach. We have derived explicit partial-wave representations for all force components in terms of the associated efficiency factors, as well as for the stiffness components. The two main effects that had not been included in the MDSA+ theory, reverberation and the evanescent wave contribution, are thereby accounted for.

Physical estimates for the rate of convergence of the iteration series, based on comparison with an equivalent interferometer with parallel plane interfaces, indicate that it should converge fast, because the relative refractive indexes are close to 1 . This is confirmed by numerical results, according to which inclusion of just the first term already yields three-digit precision. The back reflection described by this first term interferes with the incident laser light, leading to a standing wave with a period of half the wavelength in water.

Numerical plots of the axial trap stiffness per unit power as a function of objective displacement for different objective numerical apertures are given, including a typical setup, comparing situations in which reverberation and the effects of evanescent waves are included or excluded, to provide insight into the roles of these effects. The results are in good agreement with experimental data reported in [15].

In consideration of the recent interest in axial optical trapping, we have also discussed the possible appearance of nonlinear effects around the paraxial focus, when its location is close to the glass slide.

In summary, the results of the present work complement those given in [10]. They complete the theoretical basis for the absolute calibration of OT forces, opening the way for applications which require trapping close to the glass slide, as for instance when probing surface forces.

\section{ACKNOWLEDGMENTS}

This work was supported by the Brazilian agencies CNPq, FAPERJ, and INCT Fluidos Complexos. R.S.D. thanks the DTU Stochastic Systems and Signals group for its hospitality and the Brazilian government program Science Without Borders for its support.

\section{APPENDIX A: SERIES OF MULTIPLE REFLECTIONS}

From (15) we can obtain the first-order correction to the incident zero-order Debye potential (10). Expanding (15) in multipoles, performing the azimuthal integration and repeating 
the steps of Sec. II, we obtain the following expressions in terms of partial-wave (multipole) sums over the integer variables: $J=1,2, \ldots$ [eigenvalues $J(J+1)$ of the total angular momentum $J^{2}$ ] and $M=-J, \ldots, J$ (eigenvalues of $J_{z}$ ), representing the incident and scattered Debye potentials in $n$th order of iteration:

$$
\Pi_{\mathrm{in}}^{\mathrm{E}}(r, \theta, \phi)=\frac{i E_{0}}{k_{\mathrm{w}}} \sum_{J M}\left(\gamma_{J M}+\sum_{k=1}^{\infty} \delta F_{J M}^{\mathrm{E}(k)}\right) j_{J}\left(k_{\mathrm{w}} r\right) Y_{J M}(\theta, \phi)
$$

and

$$
\Pi_{\mathrm{s}}^{\mathrm{E}}(r, \theta, \phi)=-\frac{i E_{0}}{k_{\mathrm{w}}} \sum_{J M} a_{J}\left(\gamma_{J M}+\sum_{k=1}^{\infty} \delta F_{J M}^{\mathrm{E}(k)}\right) h_{J}^{(1)}\left(k_{\mathrm{w}} r\right) Y_{J M}(\theta, \phi) .
$$

We find similar expressions for the magnetic multipoles. The $\gamma_{J M}$ function representing the zero-order amplitude of the multipole expansion is given by

$$
\gamma_{J M}=2 \pi(i)^{J}(-i)^{M-1} e^{-i(M-1) \varphi_{\mathrm{C}}} \sqrt{\frac{4 \pi(2 J+1)}{J(J+1)}} G_{J M}\left(\rho_{\mathrm{C}}, \phi_{\mathrm{C}}, z_{\mathrm{C}}\right),
$$

with multipole coefficients given by [10]

$$
G_{J M}\left(\rho_{C}, \phi_{C}, z_{C}\right)=\int_{0}^{\vartheta_{m}} d \vartheta \sin \vartheta \sqrt{\cos \vartheta} e^{-\gamma^{2} \sin ^{2} \vartheta} T(\vartheta) d_{M, 1}^{J}\left(\vartheta_{\mathrm{w}}\right) g_{M}\left(\rho_{\mathrm{C}}, \phi_{\mathrm{C}}, \vartheta\right) e^{i\left[\Phi_{\mathrm{g}-\mathrm{w}}(\vartheta)+\Psi_{\mathrm{add}}(\vartheta)+k_{\mathrm{w}} \cos \vartheta_{\mathrm{w}} z_{\mathrm{C}}\right]}
$$

where

$$
\Psi_{\text {add }}(\vartheta)=2 \pi A_{\text {sa }}\left(\frac{\sin \vartheta}{\sin \vartheta_{m}}\right)^{4}+\pi A_{\text {ast }}\left(\frac{\sin \vartheta}{\sin \vartheta_{m}}\right)^{2}
$$

accounts for additional spherical aberration, corresponding to the Seidel spherical aberration parameter $A_{\text {sa }}$, and a residual field curvature coming from the Seidel astigmatism, characterized by the Seidel astigmatism parameter $A_{\text {ast }}$ and direction $\varphi_{\text {ast }}$. The anisotropy introduced by astigmatism is contained in the function

$$
g_{M}\left(\rho_{C}, \phi_{C}, \vartheta\right)=\sum_{s=-\infty}^{\infty}(-i)^{s} J_{\mathrm{s}}\left(\pi A_{\mathrm{ast}} \frac{\sin ^{2} \vartheta}{\sin ^{2} \vartheta_{m}}\right) J_{2 s+M-1}\left(k \rho_{\mathrm{C}} \sin \vartheta\right) e^{2 i s\left(\varphi_{\mathrm{ast}}-\phi_{\mathrm{C}}\right)} .
$$

The general term of the $k$ summation is given by the following recursive relation:

$$
\delta F_{J M}^{\mathrm{E}(k)}=\frac{1}{8 \pi} \sum_{J^{\prime}} \sqrt{\frac{J^{\prime}\left(J^{\prime}+1\right)\left(2 J^{\prime}+1\right)}{4 \pi}}(i)^{-J^{\prime}}\left\{\left(a_{J^{\prime}} \delta F_{J^{\prime} M}^{\mathrm{E}(k-1)}-b_{J^{\prime}} \delta F_{J^{\prime} M}^{\mathrm{M}(k-1)}\right) \tilde{\gamma}_{J, M, J^{\prime}}^{\mathrm{E}(+1)}-\left(a_{J^{\prime}} \delta F_{J^{\prime} M}^{\mathrm{E}(k-1)}+b_{J^{\prime}} \delta F_{J^{\prime} M}^{\mathrm{M}(k-1)}\right) \tilde{\gamma}_{J, M, J^{\prime}}^{\mathrm{E}(-1)}\right\}
$$

with the magnetic term given by

$$
\delta F_{J M}^{\mathrm{M}(k)}=\frac{1}{8 \pi} \sum_{J^{\prime}} \sqrt{\frac{J^{\prime}\left(J^{\prime}+1\right)\left(2 J^{\prime}+1\right)}{4 \pi}}(i)^{-J^{\prime}}\left\{\left(a_{J^{\prime}} \delta F_{J^{\prime} M}^{\mathrm{E}(k-1)}-b_{J^{\prime}} \delta F_{J^{\prime} M}^{\mathrm{M}(k-1)}\right) \tilde{\gamma}_{J, M, J^{\prime}}^{\mathrm{M}(+1)}-\left(a_{J^{\prime}} \delta F_{J^{\prime} M}^{\mathrm{E}(k-1)}+b_{J^{\prime}} \delta F_{J^{\prime} M}^{\mathrm{M}(k-1)}\right) \tilde{\gamma}_{J, M, J^{\prime}}^{\mathrm{M}(-1)}\right\} .
$$

The zero-order term is

$$
\delta F_{J M}^{\mathrm{E}(0)}=\delta F_{J M}^{\mathrm{M}(0)}=\gamma_{J M} .
$$

The $\tilde{\gamma}$ coefficients for the plane-wave superposition are given by

$$
\begin{aligned}
\tilde{\gamma}_{J, M, J^{\prime}}^{\mathrm{E}( \pm 1)}= & 2 \pi \sqrt{\frac{4 \pi(2 J+1)}{J(J+1)}}(i)^{J} \int_{C} d \alpha \sin \alpha\left\{\left[r_{\mathrm{TM}}(\pi-\alpha) \mp r_{\mathrm{TE}}(\pi-\alpha)\right] d_{M,+1}^{J}(\pi-\alpha) d_{M, \mp 1}^{J^{\prime}}(\alpha)\right. \\
& \left.-\left[r_{\mathrm{TM}}(\pi-\alpha) \pm r_{\mathrm{TE}}(\pi-\alpha)\right] d_{M,-1}^{J}(\pi-\alpha) d_{M, \mp 1}^{J^{\prime}}(\alpha)\right\} e^{-i 2 k_{\mathrm{w}} L_{\mathrm{C}} \cos \alpha}
\end{aligned}
$$

and

$$
\begin{aligned}
\tilde{\gamma}_{J, M, J^{\prime}}^{\mathrm{M}( \pm 1)}= & 2 \pi \sqrt{\frac{4 \pi(2 J+1)}{J(J+1)}}(i)^{J} \int_{C} d \alpha \sin \alpha\left\{\left[r_{\mathrm{TM}}(\pi-\alpha) \pm r_{\mathrm{TE}}(\pi-\alpha)\right] d_{M,+1}^{J}(\pi-\alpha) d_{M, \mp 1}^{J^{\prime}}(\alpha)\right. \\
& \left.+\left[r_{\mathrm{TM}}(\pi-\alpha) \mp r_{\mathrm{TE}}(\pi-\alpha)\right] d_{M,-1}^{J}(\pi-\alpha) d_{M, \mp 1}^{J^{\prime}}(\alpha)\right\} e^{-i 2 k_{\mathrm{w}} L_{\mathrm{C}} \cos \alpha} .
\end{aligned}
$$


Finally, the incident and scattered electric fields (16) and (17) in the multiple-reflection series $\sum_{k}$ are obtained from the Debye potentials by applying the real-space vector operators $\mathbf{L}=-i \mathbf{r} \times \nabla$ and $\nabla \times \mathbf{L}$, resulting in an exact expression representing the correction for the incident field in terms of the plane-waves expansion

$$
\begin{aligned}
\delta \mathbf{E}_{\mathrm{in}}(\mathbf{r})= & -\frac{E_{0}}{4 \pi} \sum_{J M k} i^{-J} \sqrt{\frac{J(J+1)(2 J+1)}{4 \pi}} \int_{0}^{2 \pi} d \beta \int_{C} d \alpha \sin \alpha e^{i M \beta} e^{-2 i k_{\mathrm{w}} L_{\mathrm{C}} \cos \alpha}\left[\left(b_{J} \delta F_{J M}^{\mathrm{M}(k-1)}+a_{J} \delta F_{J M}^{\mathrm{E}(k-1)}\right)\right. \\
& \left.\times\left(r_{\mathrm{TM}} \hat{\vartheta}+i r_{\mathrm{TE}} \hat{\varphi}\right) d_{M, 1}^{J}(\alpha)+\left(b_{J} \delta F_{J M}^{\mathrm{M}(k-1)}-a_{J} \delta F_{J M}^{\mathrm{E}(k-1)}\right)\left(r_{\mathrm{TM}} \hat{\vartheta}-i r_{\mathrm{TE}} \hat{\varphi}\right) d_{M,-1}^{J}(\alpha)\right] e^{i \mathbf{k}_{\mathrm{w}}(\pi-\alpha, \beta) \cdot \mathbf{r}} .
\end{aligned}
$$

\section{APPENDIX B: DIMENSIONLESS OPTICAL FORCE EFFICIENCY}

In this appendix we present the efficiency factor terms in the multiple reflection series. We define the filling factor $A$ representing the fraction of the laser beam power transmitted through the objective aperture and the glass slide [11]:

$$
A=16 \gamma^{2} \int_{0}^{s_{0}} d s s \exp \left(-2 \gamma^{2} s^{2}\right) \frac{\sqrt{\left(1-s^{2}\right)\left(N^{2}-s^{2}\right)}}{\left(\sqrt{1-s^{2}}+\sqrt{N^{2}-s^{2}}\right)^{2}},
$$

with $s_{0}=\min \left\{N, \mathrm{NA} / n_{\mathrm{g}}\right\}$.

We write our results for the cylindrical components perpendicular to the $z$ axis in terms of the complex efficiency factor

$$
\mathbb{Q} \equiv Q_{\rho}+i Q_{\phi} .
$$

The scattering contribution, including the zero-order (MDSA) result and the reverberation correction arising from $\delta \mathbf{E}_{\mathrm{s}} \cdot \delta \mathbf{E}_{\mathrm{s}}^{*}+$ $2 \operatorname{Re}\left(\mathbf{E}_{\mathrm{s}}^{(0)} \cdot \delta \mathbf{E}_{\mathrm{s}}^{*}\right)$, is given by

$$
\begin{aligned}
\mathbb{Q}_{s}= & \frac{-i \gamma^{2}}{4 \pi^{3} A N} e^{-i \varphi_{C}} \sum_{J M}\left\{J ( J + 2 ) \sqrt { \frac { ( J + M + 2 ) ( J + M + 1 ) } { ( 2 J + 1 ) ( 2 J + 3 ) } } \sum _ { k , k ^ { \prime } = 0 } ^ { n } \left[a_{J} a_{J+1}^{*} \delta F_{J M}^{\mathrm{E}(k)} \delta F_{J+1, M+1}^{\mathrm{E}\left(k^{\prime}\right) *}+a_{J}^{*} a_{J+1} \delta F_{J,-M}^{\mathrm{E}(k) *} \delta F_{J+1,-M-1}^{\mathrm{E}\left(k^{\prime}\right)}\right.\right. \\
& \left.+b_{J} b_{J+1}^{*} \delta F_{J M}^{\mathrm{M}(k)} \delta F_{J+1, M+1}^{\mathrm{M}\left(k^{\prime}\right) *}+b_{J}^{*} b_{J+1} \delta F_{J,-M}^{\mathrm{M}(k) *} \delta F_{J+1,-M-1}^{\mathrm{M}\left(k^{\prime}\right)}\right]+i \sqrt{(J-M)(J+M+1)}\left[a_{J}^{*} b_{J} \sum_{k, k^{\prime}=0}^{n} \delta F_{J, M}^{\mathrm{M}(k)} \delta F_{J M+1}^{\mathrm{E}\left(k^{\prime}\right) *}\right. \\
& \left.\left.+a_{J} b_{J}^{*} \sum_{k, k^{\prime}=0}^{n} \delta F_{J M}^{\mathrm{E}(k)} \delta F_{J M+1}^{\mathrm{M}\left(k^{\prime}\right) *}\right]\right\} .
\end{aligned}
$$

The scattering contribution to the axial efficiency component is given by

$$
\begin{aligned}
Q_{\mathrm{s}, \mathrm{z}}= & \frac{\gamma^{2}}{2 \pi^{3} A N} \sum_{J M} \operatorname{Im}\left\{J(J+2) \sqrt{\frac{(J+1-M)(J+1+M)}{(2 J+1)(2 J+3)}} \sum_{k, k^{\prime}=0}^{n}\left[a_{J} a_{J+1}^{*} \delta F_{J M}^{\mathrm{E}(k)} \delta F_{J+1, M}^{\mathrm{E}\left(k^{\prime}\right)^{*}}+b_{J} b_{J+1}^{*} \delta F_{J M}^{\mathrm{M}(k)} \delta F_{J+1, M}^{\mathrm{M}\left(k^{\prime}\right)^{*}}\right]\right. \\
& \left.-i M a_{J}^{*} b_{J} \sum_{k, k^{\prime}=0}^{n} \delta F_{J M}^{\mathrm{M}(k)} \delta F_{J M}^{\mathrm{E}\left(k^{\prime}\right)^{*}}\right\} .
\end{aligned}
$$

As for the extinction contribution, we first write the terms arising from $\operatorname{Re}\left(\mathbf{E}_{\text {in }}^{(0)} \cdot \mathbf{E}_{\mathrm{s}}^{*}\right)$ :

$$
\begin{aligned}
\mathbb{Q}_{\mathrm{e}}^{(0)}+\delta \mathbb{Q}_{\mathrm{e}}^{\mathrm{i}}= & \frac{-i \gamma^{2}}{2 \pi^{2} A N} \sum_{J M} \sum_{k=0}^{n} \sqrt{\frac{J(J+1)(2 J+1)}{4 \pi}}\left[\left(a_{J}^{*} \delta F_{J M}^{\mathrm{E}(k)^{*}}+b_{J}^{*} \delta F_{J M}^{\mathrm{M}(k)^{*}}\right) f_{J M}\left(\varphi_{\mathrm{C}}\right) G_{J, M-1}^{+}\right. \\
& \left.+\left(a_{J} \delta F_{J M}^{\mathrm{E}(k)}+b_{J} \delta F_{J M}^{\mathrm{M}(k)}\right) f_{J M}\left(\varphi_{\mathrm{C}}\right)^{*} G_{J, M+1}^{-}{ }^{*}\right],
\end{aligned}
$$

and

$$
Q_{\mathrm{e}, z}^{(0)}+\delta Q_{\mathrm{e}, z}^{\mathrm{i}}=\frac{\gamma^{2}}{\pi^{2} A N} \operatorname{Re}\left\{\sum_{J M} \sum_{k=0}^{n} \sqrt{\frac{J(J+1)(2 J+1)}{4 \pi}}\left(a_{J}^{*} \delta F_{J M}^{\mathrm{E}(k)^{*}}+b_{J}^{*} \delta F_{J M}^{\mathrm{M}(k)^{*}}\right) f_{J M}\left(\varphi_{\mathrm{C}}\right) G_{J M}^{C}\right\},
$$

with $f_{J M}\left(\varphi_{\mathrm{C}}\right)=2 \pi(i)^{J}(-i)^{(M-1)} e^{-i(M-1) \varphi_{\mathrm{C}}}$ and the multipole coefficients

$$
G_{J M}^{ \pm}\left(\rho_{\mathrm{C}}, \phi_{\mathrm{C}}, z_{\mathrm{C}}\right)=\int_{0}^{\vartheta_{m}} d \vartheta \sin \vartheta \sin \vartheta_{\mathrm{w}} \sqrt{\cos \vartheta} e^{-\gamma^{2} \sin ^{2} \vartheta} T(\vartheta) d_{M \pm 1,1}^{J}\left(\vartheta_{\mathrm{w}}\right) g_{M}\left(\rho_{\mathrm{C}}, \phi_{\mathrm{C}}, \vartheta\right) e^{i\left[\Phi_{\mathrm{g}-\mathrm{w}}(\vartheta)+\Psi_{\mathrm{add}}(\vartheta)+k_{\mathrm{w}} \cos \vartheta_{\mathrm{w}} z_{\mathrm{C}}\right]}
$$


and

$$
G_{J M}^{C}\left(\rho_{\mathrm{C}}, \phi_{\mathrm{C}}, z_{\mathrm{C}}\right)=\int_{0}^{\vartheta_{m}} d \vartheta \sin \vartheta \cos \vartheta_{\mathrm{w}} \sqrt{\cos \vartheta} e^{-\gamma^{2} \sin ^{2} \vartheta} T(\vartheta) d_{M, 1}^{J}\left(\vartheta_{\mathrm{w}}\right) g_{M}\left(\rho_{\mathrm{C}}, \phi_{\mathrm{C}}, \vartheta\right) e^{i\left[\Phi_{\mathrm{g}-\mathrm{w}}(\vartheta)+\Psi_{\mathrm{add}}(\vartheta)+k_{\mathrm{w}} \cos \vartheta_{\mathrm{w}} z_{\mathrm{C}}\right]} .
$$

Finally, for the term associated with $\operatorname{Re}\left(\delta \mathbf{E}_{\text {in }} \cdot \mathbf{E}_{\mathrm{s}}^{*}\right)$, we have

$$
\delta \mathbb{Q}_{\mathrm{e}}^{\mathrm{ii}}=\frac{i \gamma^{2}}{64 \pi^{4} A N} \sum_{J M} \sqrt{J(J+1)(2 J+1)} \Theta_{J M}^{\perp},
$$

and

$$
\delta Q_{\mathrm{e}, z}^{\mathrm{ii}}=-\frac{\gamma^{2}}{32 \pi^{4} A N} \operatorname{Re}\left(\sum_{J M} \sqrt{J(J+1)(2 J+1)} \Theta_{J M}^{z}\right) .
$$

The functions $\Theta_{J M}^{\perp}$ and $\Theta_{J M}^{z}$ are defined by

$$
\Theta_{J M}^{\perp}=\sum_{J^{\prime}} \int_{C} d \alpha \sin ^{2} \alpha\left[e^{-2 i k_{\mathrm{w}} L_{\mathrm{C}} \cos \alpha} H_{J, J^{\prime}, M, M+1}(\alpha)+e^{2 i k_{\mathrm{w}} L_{\mathrm{C}}(\cos \alpha)^{*}} H_{J, J^{\prime}, M, M-1}(\alpha)^{*}\right],
$$

and

$$
\Theta_{J M}^{z}=\sum_{J^{\prime}} \int_{C} d \alpha \sin \alpha \cos (\pi-\alpha) e^{-i 2 k_{\mathrm{w}} L_{\mathrm{C}} \cos \alpha} H_{J, J^{\prime}, M, M}(\alpha) .
$$

The coefficients $H_{J, J^{\prime}, M, M^{\prime}}(\alpha)$ describing the interference between the fields $\delta \mathbf{E}_{\text {in }}$ and $\mathbf{E}_{\mathrm{s}}$, are given by

$$
\begin{aligned}
H_{J, J^{\prime}, M, M^{\prime}}(\alpha)= & 2 \pi \sqrt{J^{\prime}\left(J^{\prime}+1\right)\left(2 J^{\prime}+1\right)}(-i)^{\left(M^{\prime}-M\right)}(i)^{\left(J^{\prime}-J\right)} e^{-i\left(M^{\prime}-M\right) \varphi_{C}}\left[(-i) B_{J M}\left(\gamma_{J^{\prime}, M^{\prime}}^{\mathrm{E}^{*}} a_{J^{\prime}}^{*} r_{\mathrm{TM}}-\gamma_{J^{\prime}, M^{\prime}}^{\mathrm{M}^{*}} b_{J^{\prime}}^{*} r_{\mathrm{TE}}\right)\right. \\
& \times\left[d_{M^{\prime}, 1}^{J^{\prime}}(\pi-\alpha)-d_{M^{\prime},-1}^{J^{\prime}}(\pi-\alpha)\right] d_{M,-1}^{J}(\alpha)+(i) B_{J M}\left(\gamma_{J^{\prime}, M^{\prime}}^{\mathrm{E}^{*}} a_{J^{\prime}}^{*} r_{\mathrm{TE}}-\gamma_{J^{\prime}, M^{\prime}}^{\mathrm{M}^{*}}, b_{J^{\prime}}^{*} r_{\mathrm{TM}}\right) \\
& \times\left[d_{M^{\prime}, 1}^{J^{\prime}}(\pi-\alpha)+d_{M^{\prime},-1}^{J^{\prime}}(\pi-\alpha)\right] d_{M,-1}^{J}(\alpha)+(-i) A_{J M}\left(\gamma_{J^{\prime}, M^{\prime}}^{\mathrm{E}^{*}} a_{J^{\prime}}^{*} r_{\mathrm{TM}}+\gamma_{J^{\prime}, M^{\prime}}^{\mathrm{M}^{*}}, b_{J^{\prime}}^{*} r_{\mathrm{TE}}\right) \\
& \times\left[d_{M^{\prime}, 1}^{J^{\prime}}(\pi-\alpha)-d_{M^{\prime},-1}^{J^{\prime}}(\pi-\alpha)\right] d_{M, 1}^{J}(\alpha)+(-i) A_{J M}\left(\gamma_{J^{\prime}, M^{\prime}}^{\mathrm{E}^{*}} a_{J^{\prime}}^{*}, r_{\mathrm{TE}}+\gamma_{J^{\prime}, M^{\prime}}^{\mathrm{M}^{*}}, b_{J^{\prime}}^{*}, r_{\mathrm{TM}}\right) \\
& \left.\times\left[d_{M^{\prime}, 1}^{J^{\prime}}(\pi-\alpha)+d_{M^{\prime},-1}^{J^{\prime}}(\pi-\alpha)\right] d_{M, 1}^{J}(\alpha)\right],
\end{aligned}
$$

with

$$
\begin{gathered}
A_{J M}=i \sum_{k=0}^{n-1}\left(a_{J} \delta F_{J M}^{\mathrm{E}(k)}+b_{J} \delta F_{J M}^{\mathrm{M}(k)}\right), \\
B_{J M}=-i \sum_{k=0}^{n-1}\left(a_{J} \delta F_{J M}^{\mathrm{E}(k)}-b_{J} \delta F_{J M}^{\mathrm{M}(k)}\right), \\
\gamma_{J M}^{\mathrm{E}}=\sum_{k=0}^{n} \delta F_{J M}^{\mathrm{E}(k)}
\end{gathered}
$$

and

$$
\gamma_{J M}^{\mathrm{M}}=\sum_{k=0}^{n} \delta F_{J M}^{\mathrm{M}(k)}
$$

[1] A. Ashkin, Optical Trapping and Manipulation of Neutral Particles Using Lasers: A Reprint Volume With Commentaries (World Scientific, Singapore, 2006).

[2] E. Schäffer, S. F. Nørrelykke, and J. Howard, Langmuir 23, 3654 (2007).

[3] C. Gutsche, U. F. Keyser, K. Kegler, F. Kremer, and P. Linse, Phys. Rev. E 76, 031403 (2007).

[4] D. S. Ether, Jr. et al., Europhys. Lett. 112, 44001 (2015).

[5] B. Pontes, N. B. Viana, L. T. Salgado, M. Farina, V. M. Neto, and H. M. Nussenzveig, Biophys. J. 101, 43 (2011).
[6] K. Berg-Sørensen and H. Flyvbjerg, Rev. Sci. Instrum. 75, 594 (2004).

[7] S. F. Tolic-Nørrelykke, E. Schäffer, J. Howard, F. S. Pavone, F. Jülicher, and H. Flyvbjerg, Rev. Sci. Instrum. 77, 103101 (2006).

[8] S. F. Nørrelykke and H. Flyvbjerg, Rev. Sci. Instrum. 81, 075103 (2010).

[9] S. F. Nørrelykke and H. Flyvbjerg, Phys. Rev. E 83, 041103 (2011).

[10] R. S. Dutra, N. B. Viana, P. A. Maia Neto, and H. M. Nussenzveig, Phys. Rev. A 90, 013825 (2014). 
[11] N. B. Viana, M. S. Rocha, O. N. Mesquita, A. Mazolli, P. A. Maia Neto, and H. M. Nussenzveig, Phys. Rev. E 75, 021914 (2007).

[12] A. A. R. Neves, A. Fontes, C. L. Cesar, A. Camposeo, R. Cingolani, and D. Pisignano, Phys. Rev. E 76, 061917 (2007).

[13] R. S. Dutra, N. B. Viana, P. A. Maia Neto, and H. M. Nussenzveig, J. Opt. A 9, S221 (2007).

[14] K. C. Neuman, E. A. Abbondanzieri, and S. M. Block, Opt. Lett. 30, 1318 (2005).

[15] A. H. Mack, D. J. Schlingman, L. Regan, and S. G. J. Mochrie, Rev. Sci. Instrum. 83, 103106 (2012).

[16] R. Pollari and J. N. Milstein, Opt. Express 23, 28857 (2015).

[17] G. Mie, Ann. Phys. (Berlin) 25, 377 (1908).

[18] P. Debye, Ann. Phys. (Berlin) 30, 755 (1909).

[19] B. Richards and E. Wolf, Proc. R. Soc. London A 253, 358 (1959).

[20] P. A. Bobbert and J. Vlieger, Physica A 137, 209 (1986).

[21] H. Weyl, Ann. Phys. (Berlin) 60, 481 (1919).

[22] J. A. Stratton, in Electromagnetic Theory (McGraw-Hill, New York, 1941), pp. 392-423.

[23] G. C. Sherman, J. A. Devy, and L. Mandel, Opt. Commun. 6, 115 (1972).

[24] H. M. Nussenzveig, in Optics in Four Dimensions-1980, edited by M. A. Machado and L. M. Narducci, AIP Conf. Proc. No. 65 (AIP, New York, 1980), p. 9.

[25] G. Videen, J. Opt. Soc. Am. A 8, 483 (1991).
[26] T. Wriedt and A. Doicu, Opt. Commun. 152, 376 (1998).

[27] S. Chang, J. T. Kim, J. H. Jo, and S. S. Lee, Opt. Commun. 139, 252 (1997).

[28] J. Xu, W.-P. Zang, and J.-G. Tian, Opt. Express 23, 4195 (2015).

[29] A. Sommerfeld, Optics (Academic, New York, 1954), p. 47.

[30] J. P. Barton, W. Ma, S. A. Schaub, and D. R. Alexander, Appl. Opt. 30, 4706 (1991).

[31] W. Itami and Y. Kawata, J. Appl. Phys. 89, 5876 (2001).

[32] P. Török, P. Varga, Z. Laczik, and G. R. Booker, J. Opt. Soc. Am. A 12, 325 (1995).

[33] A. J. Devaney and E. Wolf, J. Math. Phys. 15, 234 (1974).

[34] C. J. Bouwkamp and H. B. G. Casimir, Physica 20, 539 (1954).

[35] M. Abramowitz and I. Stegun, Handbook of Mathematical Functions (Dover, New York, 1972).

[36] C. F. Bohren and D. R. Huffman, Absorption and Scattering of Light by Small Particles (Wiley, New York, 1983), Chap. 4.

[37] A. R. Edmonds, Angular Momentum in Quantum Mechanics (Princeton University Press, Princeton, 1957).

[38] See Supplemental Material at http://link.aps.org/supplemental/ 10.1103/PhysRevA.94.053848 for a Mathematica ${ }^{\circledR}$ notebook file that calculates the axial force from the analytical expressions presented here.

[39] S. Yehoshua, R. Pollari, and J. N. Milstein, Biophys. J. 108, 2759 (2015). 